\title{
Pancreas transplantation
}

\author{
SA WHITE, DW MANAS
}

\section{Department of Hepatobiliary and Transplant Surgery, The Freeman Hospital, Newcastle Upon} Tyne, UK

\section{ABSTRACT}

Over the last 5 years, there has been a resumed interest in treating diabetes by transplantation, particularly islet transplantation. However, despite advances being reported in Canada and the US, replication in the UK has been much more difficult. At present there is still only one treatment that can consistently reverse insulin independence in the long-term and that is whole pancreas transplantation. Long-term normoglycaemia has beneficial effects on preventing and ameliorating the secondary complications of diabetes and will be discussed.

\section{KEYWORDS}

Pancreas - Diabetes - Islets - Renal transplant

\section{CORRESPONDENCE TO}

Steve White, Consultant Hepatobiliary and Transplant Surgeon, The Freeman Hospital, High Heaton, Newcastle Upon Tyne NE7 7DN, UK E: steve.white@nuth.nhs.uk

Over the last 5 years, there has been a resumed interest in treating diabetes by transplantation, particularly islet transplantation. However, despite advances being reported in Canada (Edmonton) ${ }^{1}$ and the US (Minneapolis, ${ }^{2}$ Miami $^{3}$ ), replication in the UK has been much more difficult.

In the UK, pancreas transplantation has always been regarded as a formidable operation with a perceived misconception of significant recipient co-morbidity, high rates of re-operation, sepsis and pancreatic leaks. Per capita population, the number of patients receiving pancreas transplants has always been low compared to the US. Its value has to be balanced against the operative morbidity and mortality and the long-term sequele of the chronic immunosuppression needed to prevent graft rejection. In comparison to pancreas transplantation, the argument for islet transplantation has always been much easier because of its reduced morbidity. Pancreas transplantation has to be restricted to various patient categories with established complications or extremely labile diabetes that are considered to be more lifethreatening than the side effects of the immunosuppression and surgical morbidity. The categories are simultaneously with a kidney (SPK), pancreas after kidney (PAK) or, for non-uraemic patients, pancreas transplant alone (PTA), these patients have life-threatening hypoglycaemic unawareness.

The benefit of pancreas transplantation is an improved quality of life and insulin independence. SPK has been shown to prolong survival when compared to either diabetic patients receiving a kidney transplant alone (KTA) $)^{4-6}$ or versus diabetic patients with end-stage renal failure. ${ }^{7}$ Pancreas transplantation can also: (i) stabilise retinopathy; ${ }^{8,9}$ (ii) reverse the pathological changes seen in native diabetic kidneys; ${ }^{10,11}$ (iii) improve polyneuropathy; ${ }^{12}$ and (iv) decrease the progression of coronary artery disease. ${ }^{13}$

With the improved results being reported in the US, interest in the UK has steadily increased. ${ }^{14}$ In 2001, UK Transplant established a Pancreas Transplant Task force to address pertinent issues relating to indications, organ allocation and practice standards. There are now six recognised centres currently performing pancreas transplants formerly funded by NSCAG now known as the National Commissioning Group (NCG). These centres are Cambridge, Manchester, Newcastle, West London (incorporating St Mary's), Oxford, Guy's Hospital and Edinburgh funded by the Scottish Office. The NCG is a standing committee of the newly established National Specialised Services Commissioning Group (NSCG). The NSCG oversees the national commissioning of highly specialised services (e.g. pancreas and liver transplantation) and facilitates collaborative working at a specialised commissioning group level. For the first time, in 2005 over 100 pancreas transplants were performed in the UK. These comprised 102 SPK, 14 PAK and 2 PTA. ${ }^{14}$

Centres performing pancreas transplants are encouraged to report their results to the International Pancreas Transplant Registry (IPTR) based in Minneapolis. The 
latest report from the IPTR shows patient survival rates in all three categories ranging between $95-98 \%$ at 1 year and insulin independence of up to $85 \%$ at 1 year. ${ }^{15}$ Nevertheless, there has been recent controversy regarding survival advantages for solitary pancreas transplants (PTA and PAK). Venstrom et al. ${ }^{16}$ recently demonstrated higher rates of mortality at 4 years when compared to diabetic patients on the waiting list for either PTA (85\% versus $92 \%$ ) or PAK ( $84.5 \%$ versus $88 \%$ ). Nonetheless, these results have been interpreted with caution as the analysis was based on waiting list data. It is generally well recognised that waiting lists are prone to change, particularly when a patient's condition deteriorates, such that $8 \%$ of patients who were turned down for a pancreas transplant, subsequently had KTA. Nearly 10\% of patients were also listed at multiple centres. Patients who are too unwell for a transplant are removed from the waiting list thus skewing the data for any apparent survival advantage. A subsequent analysis from the IPTR and UNOS database suggests that the mortality rate for solitary pancreas transplant recipients is, in fact, less than waiting-list patients when these confounding factors are taken into consideration. ${ }^{15}$

Since 2003, the number of SPK transplants performed in the US has remained almost static but the number of solitary pancreas transplants (PAK and PTA) has quadrupled. ${ }^{17}$ This is not the case in the UK, although the rise is slowly improving (10 transplants in 2004, 16 transplants in 2005) the numbers are still small. ${ }^{14}$ There are definite incentives to performing solitary pancreas transplants. Historically, solitary pancreas transplants were disadvantaged because of the immunological deterrent of higher rates of acute rejection when compared to SPK. However, with newer immunosuppressive regimens and induction antibody therapy, rejection rates of SPK and PAK are now similar. ${ }^{18}$ Another important observation by Gruessner et al. ${ }^{17}$ is that almost $50 \%$ of potential SPK patients who wait for longer than 4 years die whilst waiting, an observation that is not consistent with PAK. This strongly emphasises the importance of the correction of uraemia and the life-saving nature of a kidney transplant as opposed to the life-improving concept of a pancreas transplant.

In the US, the number of living-donor renal transplants per annum is now beyond that of cadaver-donor renal transplants. The emphasis is now to perform a living-donor renal transplant either at the same time as a cadaver pancreas becomes available (e.g. simultaneous pancreas living kidney (SPLK) ${ }^{19}$ or before a pancreas as a conventional PAK. Either combination offers the potential benefits of a living-donor renal transplant, such as pre-emptive transplantation. In this context, recipients are generally fitter with less co-morbidity having not yet started dialysis. Indeed, pre-emptive SPK has been shown to offer a survival benefit with reduced cardiac deaths. ${ }^{20}$ Either strategy of SPLK or a livingdonor kidney with PAK offers the potential for better long-term graft survival, shorter waiting times and expands the donor pool. ${ }^{21,22}$ The only advantage of SPK or SPLK is that it can be performed as a single procedure but SPK has a higher rate of re-laparotomy when compared to PAK (26\% versus $15 \%) .{ }^{22}$ Disadvantages of PAK are worsening renal function when the pancreas is added and long-term pancreas graft loss; however, with the improvements in immunosuppression and less immunological graft loss, these disadvantages may no longer be apparent. There are also logistical problems with SPLK, for example, co-ordinating the recipient and donor surgery without compromising the donor pancreatic cold ischaemic time. Early experience suggests that this is not a major drawback.

\section{Conclusions}

Taken together, there is now a need to consolidate the experience and further improve upon the results of pancreas transplantation in the UK. This should be done by changing the emphasis to optimise the use of livingdonor renal transplants with PAK and by performing pre-emptive transplants whenever possible.

\section{References}

1. Ryan EA, Paty BW, Senior PA, Bigam D, Alfadhli E, Kneteman NM et al. Five year follow up after clinical islet transplantation. Diabetes 2005; 54: 2060-9.

2. Hering BJ, Kandaswamy R, Ansite JD, Eckman PM, Nakano M, Sawada T et al. Single donor, marginal dose islet transplantation with type 1 diabetes. JAMA 2005; 293: 830-5.

3. Froud T, Ricordi C, Baidal DA, Hafiz MM, Ponte G, Cure P et al. Islet transplantation in type 1 diabetes mellitus using cultured islets and steroid free immunosuppression: Miami experience. Am J Transplant 2005; 5: 2037-46.

4. Tyden G, Bolinder J, Solders G, Brattstrom C, Tibell A, Groth CG. Improved survival in patients with insulin dependent diabetes mellitus and end stage diabetic nephropathy 10 years after combined pancreas and kidney transplantation. Transplant 1999; 67: 645-8.

5. Reddy KS, Stablein D, Taranto S, Stratta RJ, Johnston TD, Waid TH et al. Long-term survival following simultaneous kidney pancreas transplantation versus kidney transplantation alone in patients with type 1 diabetes and renal failure. Am J Kidney Dis 2003; 41: 464-70.

6. Ojo AO, Meier-Kriesche HU, Hanson JA, Leichtman A, Magee JC, Cibrik D et al. Impact of simultaneous pancreas kidney transplantation on long-term patient survival. Transplantation 2001; 71: 82-90.

7. Smets YF, Westendorp RG, van der Pijl JW, de Charro FT, Ringers J, de Fijter JW et al. Effect of simultaneous pancreas-kidney transplantation on mortality of patients with type- 1 diabetes mellitus and end-stage renal failure. Lancet 1999; 353: 1915-9.

8. Wang Q, Klein R, Moss SE, Klein BE, Hoyer C, Barke $\mathrm{K}$ et al. The influence of 
combined kidney-pancreas transplantation on the progression of diabetic retinopathy. A case series. Ophthalmology 1994; 101: 1071-6.

9. Koznarova R, Saudek F, Sosna T, Adamec M, Jedinakova T, Boucek P et al. Beneficial effect of pancreas and kidney transplantation on advanced diabetic retinopathy. Cell Transplant 2000; 9: 903-8.

10. Fioretto P, Steffes MW, Sutherland DER, Goetz FC, Mauer M. Reversal of lesions of diabetic nephropathy after pancreas transplantation. N Eng/ J Med 1998; 339: 69-75.

11. Fioretto $P$, Sutherland DER, Najafian B, Mauer M. Remodelling of renal interstitial and tubular lesions in pancreas transplant recipients. Kidney Int 2006; 69 : 907-12.

12. Kennedy WR, Navarro X, Goetz FC, Sutherland DE, Najarian JS. Effects of pancreatic transplantation on diabetic neuropathy. N Engl J Med 1990; 322: 1031-7.

13. Jukema JW, Smets YFC, van der Pijl JW, Zwinderman AH, Vliegen HW, Ringers $J$ et al. Impact of simultaneous pancreas and kidney transplantation on progression of coronary atherosclerosis in patients with end stage renal failure due to type 1 diabetes. Diabetes Care 2002; 25: 906-11.

14. UK Transplant. Transplant Statistics. <www.uktransplant.org> [Accessed July 2006].

15. Gruessner AC, Sutherland DER. Pancreas transplant outcomes for United States (US) and non US cases as reported to the United Network for Organ Sharing (UNOS) and the International Pancreas Transplant Registry (IPTR) as of
June 2004. Clin Transplant 2005; 19: 433-55.

16. Venstrom JM, McBride MA, Rother KI, Hirshberg B, Orchard TJ, Harlan DM. Survival after pancreas transplantation in patients with diabetes and preserved kidney function. JAMA 2003; 290: 2817-23.

17. Gruessner RWG, Sutherland DER, Gruessner AC. Mortality assessment for pancreas transplants. Am J Transplant 2004; 4: 2018-26.

18. Larson TS, Bohorquez H, Rea DJ, Nyberg SL, Prieto M, Sterioff S et al. Pancreas after kidney transplantation: an increasingly attractive alternative to SPK transplantation. Transplantation 2004; 77: 838-43.

19. Farney AC, Cho E, Schwitzer EJ, Dunkin B, Philosophe B, Colonna J et al Simultaneous cadaver pancreas living donor kidney transplantation: a new approach for the type 1 diabetic uremic patient. Ann Surg 2000; 232: 696-703.

20. Pruijm MT, de Fijter HJ, Doxiadis II, Vandenbroucke JP. Pre-emptive versus non-preemptive simultaneous pancreas-kidney transplantation: a single-center, long-term follow up study. Transplantation 2006; 81: 1119-24.

21. Larson TS, Bohorquez H, Rea DJ, Nyberg SL, Prieto M, Sterioff S et al. Pancreas after kidney transplantation: an increasingly attractive alternative to simultaneous pancreas kidney transplantation. Transplantation 2004; 77 : 838-43.

22. Humar A, Ramcharan $T$, Kandaswamy R, Matas A, Gruessner RW, Gruessner AC et al. Pancreas after kidney transplants. Am J Surg 2001; 182: 155-61.

\section{e-Letters - new additions}

Since the last issue of the Annals, the following letters have been published on our website $<$ http://www.rcseng.ac.uk/publications/eletters/>:

Is describing fractures a dying art? -2 responses

A multicentre audit of single-use surgical instruments (SUSI) for tonsillectomy and adenoidectomy - 2 responses

Pancreas-sparing distal duodenectomy for infra-papillary neoplasms -2 responses

Reducing diagnostic errors in musculoskeletal trauma by reviewing non-admission orthopaedic referrals in the next-day trauma meeting -2 responses

Simple and safe technique of port closure -2 responses

Diagnostic fine-needle aspiration in postoperative wound infections is more accurate at predicting causative organisms than wound swabs -1 response 